\title{
Downregulation of long non-coding RNA NR2F2-AS1 inhibits proliferation and induces apoptosis of nasopharyngeal carcinoma cells by upregulating the expression of PTEN
}

\author{
$\mathrm{HE} \mathrm{QIN}^{1}$ and CHONG QIN ${ }^{2}$ \\ ${ }^{1}$ Department of Otolaryngology, Head and Neck Surgery, Beijing Tsinghua Changgung Hospital, \\ School of Clinical Medicine, Tsinghua University, Changping, Beijing 102218; ${ }^{2}$ Department of Emergency, \\ Dongying Shengli Hospital, Dongying, Shandong 257055, P.R. China
}

Received April 22, 2019; Accepted October 22, 2019

DOI: $10.3892 / \mathrm{ol} .2019 .11211$

\begin{abstract}
The novel long non-coding RNA NR2F2-AS1 has been characterized as an oncogene in lung cancer. The present study aimed to investigate the role of NR2F2-AS1 in nasopharyngeal carcinoma (NPC). The results demonstrated that expression of NR2F2-AS1 and phosphatase and tensin homolog (PTEN) were negatively associated with each other in NPC tissues. Furthermore, upregulated NR2F2-AS1 expression levels and downregulated PTEN expression levels in NPC tissues predicted less favorable survival outcomes in patients with NPC. Transfection of NPC cells with NR2F2-AS1 small interfering RNA resulted in increased expression of PTEN. In addition, NR2F2-AS1 silencing and PTEN overexpression resulted in decreased proliferation and an increase in the apoptotic rate of NPC cells. In conclusion, NR2F2-AS1 downregulation decreased proliferation and increased apoptosis of NPC cells via upregulation of PTEN.
\end{abstract}

\section{Introduction}

Nasopharyngeal carcinoma (NPC) is a subtype of head and neck malignancy that develops from the lining of the nasopharynx (1). The incidence of NPC varies a lot across different regions of the world, with a high prevalence in Southeast Asia, North Africa and Southern China (2). Patients with NPC are prone to develop neck invasion and distant tumor metastases $(3,4)$. Although radiotherapy and chemotherapy have been used to treat advanced NPC, effective therapeutic options remain limited (5). Numerous risk factors, including

Correspondence to: Dr He Qin, Department of Otolaryngology, Head and Neck Surgery, Beijing Tsinghua Changgung Hospital, School of Clinical Medicine, Tsinghua University, 168 Litang Road, Changping, Beijing 102218, P.R. China

E-mail: heqin007@126.com

Key words: nasopharyngeal carcinoma, long non-coding RNA NR2F2-AS1, phosphatase and tensin homolog, prognosis
Epstein-Barr virus infections, have been identified as risk factors of NPC (6); however, the pathogenesis of NPC remains unclear, which limits the development of novel therapeutic approaches. The 5-year survival rate of patients with advanced NPC was $<50 \%$ in 2016 worldwide $(1,2)$.

Phosphatase and tensin homolog (PTEN) is a tumor suppressor that has been validated in the majority of mammals (7). Mutations in the PTEN gene are associated with numerous types of cancer, such as endometrial cancer (7). PTEN regulates the cell cycle and inhibits the accelerated proliferation of cancer cells by inhibiting the PI3K/AKT survival pathway $(8,9)$. Activation of PTEN may therefore be a promising approach for cancer prevention and treatment (10). It has been reported that PTEN signaling in cancer is regulated by long non-coding (lnc)RNAs (11), which are defined as functional RNA transcripts $>200$ nucleotides in length with no protein-coding capacity (12). LncRNAs participate in cancer biology by regulating the expression of oncogenes or tumor suppressors to affect cancer cell behaviors, such as proliferation and apoptosis (12). A recent study by Zhang et al (13) reported that the novel oncogenic IncRNA, NR2F2-AS1, interacts with the microRNA (miR) miR-320b by serving as its endogenous sponge in lung cancer. Furthermore, miR-320b and PTEN can upregulate each other, affecting cancer biology (14). The present study therefore aimed to investigate whether NR2F2-AS1 could interact with PTEN.

\section{Materials and methods}

Patients with NPC and follow-up. In the present study, 58 patients with NPC (22 men and 18 women; age range, 32-66 years; mean age, $44 \pm 12.3$ years) were selected among the 101 patients with NPC who were admitted at the Dongying Shengli Hospital between June 2010 and November 2013. Only patients newly diagnosed with NPC and who completed the 5-year follow-up (via phone call every month) following admission were included. The exclusion criteria were as follows: i) Patients diagnosed with clinical disorders other than NPC; ii) patients with recurrent NPC; iii) patients who had started receiving therapy for any clinical disorders; iv) patients who died of causes unrelated to NPC during follow-up; and 
vi) patients with undetectable PTEN in tumor tissues. Based on the clinical data, 10,12,18 and 18 patients had NPC at clinical stages I, II, III and IV, respectively, according to the eighth edition of the American Joint Committee on Cancer staging system (15). This study was approved by the Ethics Committee of Dongying Shengli Hospital, and signed informed consent was provided by all patients.

NPC tissues and cells. Patients were diagnosed by histopathological analysis of tumor biopsy. During the biopsy, non-tumor tissues ( $2 \mathrm{~cm}$ adjacent to tumors) and NPC tissues (0.013-0.019 g per sample) were collected from each patient with NPC. Samples were stored at $-80^{\circ} \mathrm{C}$ before use. All tissues were histopathologically confirmed. The two NPC cell lines C666-1 and 13-9B (SHUNRAN) cells were used. Cells were cultured in RPMI-1640 medium (Sigma-Aldrich; Merck KGaA) supplemented with $10 \%$ fetal bovine serum (FBS; Sigma-Aldrich; Merck $\mathrm{KGaA}$ ) and placed at $37^{\circ} \mathrm{C}$ in a humidified incubator containing $5 \% \mathrm{CO}_{2}$.

Transient transfections. Negative control small interfering (si)RNA (5'-AUUGUCGUACGUAGCUACGUA-3'), NR2F2-AS1 SiRNA (5'-GCUUCUCUCUUGAUUACA UUG-3') and PTEN siRNA (5'-AAAUCUAGGGCAUCU UGUGCC-3') were provided by Sangon Biotech Co., Ltd. pcDNA3 vector expression PTEN and empty pcDNA3 vector were provided by GenePharma Co. Ltd. C666-1 and 13-9B cells were harvested when confluence reached 70-80\%. A total of $1 \times 10^{5}$ cells were transfected with $10 \mathrm{nM}$ negative control siRNA (negative control, NC), 10 nM NR2F2-AS1 siRNA or PTEN siRNA, 12 nM pcDNA3 vector expression PTEN or $12 \mathrm{nM}$ empty pcDNA3 vector using Lipofectamine ${ }^{\circledR}$ 2000 reagent (Invitrogen; Thermo Fisher Scientific, Inc.). Untransfected cells were used as a control (C) as well as cells transfected with the empty vector (NC). Cells were transfected for $24 \mathrm{~h}$ before performing subsequent experiments.

Reverse transcription-quantitative polymerase chain reaction $(R T-q P C R)$. NPC and non-tumor frozen tissues were powdered by grinding in liquid nitrogen. TRIzol ${ }^{\circledR}$ reagent (Invitrogen; Thermo Fisher Scientific, Inc.) was mixed with tissue powder (0.5 ml per $0.01 \mathrm{~g}$ tissue) or C666-1 and 13-9B cells $(0.5 \mathrm{ml}$ per $1 \times 10^{5}$ cells) to extract total RNAs. All RNA samples were digested with DNase I to remove genomic DNA. cDNA was generated using QuantiTect Reverse Transcription kit (Qiagen China Co., Ltd.) according to the manufacturer's protocol. QuantiFast SYBR Green PCR kit (Qiagen China Co., Ltd.) was used to prepare all PCR mixtures, and 18S rRNA was used as an endogenous control. PCR mixture $(20 \mu \mathrm{l})$ contained $0.5 \mathrm{nM}$ of each primer and $1 \mu \mathrm{l}$ template DNA. The thermocycling conditions of the RT-qPCR were as follows: $95^{\circ} \mathrm{C}$ for $30 \mathrm{sec}$; followed by 40 cycles of $95^{\circ} \mathrm{C}$ for $10 \mathrm{sec}$ and $58^{\circ} \mathrm{C}$ for $45 \mathrm{sec}$. Each sample was analyzed at least three times. The relative expressions levels were normalized to endogenous control and expression was calculated using the $2^{-\Delta \Delta \mathrm{Cq}}$ method (16). The sample with the lowest expression level was set to a value of ' 1 ' and all other samples were normalized to this sample. The sequences of the primers were as follows: NR2F2-AS1 forward, 5'-TCAGCCGGAAAACTACAAGCTC-3' and reverse, 5'-CTTCGTGTAGCTGTTCCACC-3'; 18s forward,
5'-GTAACCCGTTGAACCCCATT-3' and reverse, 5'-CCA TCCAATCGGTAGTAGCG-3'; and PTEN forward, 5'-TTG GCGGTGTCATAATGTC-3' and reverse, 5'-CAGAAAGAC TTGAAGGCGTA-3'.

Cell proliferation assay. At $24 \mathrm{~h}$ post-transfection, C666-1 and $13-9 \mathrm{~B}$ cells were harvested using $0.25 \%$ trypsin. A total of $4 \times 10^{4}$ cells were mixed with $1 \mathrm{ml}$ RPMI-1640 medium containing $10 \%$ FBS to prepare single-cell suspensions. Cells were seeded in 96 -well plates $(0.1 \mathrm{ml}$ per well) and cultured at $37^{\circ} \mathrm{C}$ and $5 \% \mathrm{CO}_{2}$. Subsequently, to detect cell proliferation, Cell Counting Kit-8 solution (10 $\mu \mathrm{l}$; Dojindo Molecular Technologies, Inc.) was added in each well at $4 \mathrm{~h}$ before cell collection. Cells were collected every $24 \mathrm{~h}$ until $96 \mathrm{~h}$. Absorbance was measured at $450 \mathrm{~nm}$ using a microplate reader.

Cell apoptosis assay. At $24 \mathrm{~h}$ post-transfection, C666-1 and 13-9B cells were harvested using $0.25 \%$ trypsin. A total of $4 \times 10^{4}$ cells were mixed with $1 \mathrm{ml}$ serum-free RPMI-1640 medium to prepare single-cell suspensions. Cells were seeded in 6-well plates $\left(2 \mathrm{ml}\right.$ per well) and cultured at $37^{\circ} \mathrm{C}$ and $5 \%$ $\mathrm{CO}_{2}$ for $48 \mathrm{~h}$. Cells were then harvested with $0.25 \%$ trypsin, mixed with fresh cell culture medium and incubated with propidium iodide (cat. no. AD01-02; Dojindo Molecular Technologies, Inc.) and annexin V-fluorescein isothiocyanate (cat. no. AD02-05; Dojindo Molecular Technologies, Inc.) at $4^{\circ} \mathrm{C}$ for $20 \mathrm{~min}$ in the dark. All steps were performed according to manufacturer's instructions. Apoptotic cells were subsequently analyzed using a flow cytometer using BD FACSCalibur Flow Cytometer (BD Biosciences). Data were analyzed by CytExpert v.2.3 flow cytometry software (Beckman Coulter, Inc.).

Western blotting. At $24 \mathrm{~h}$ post-transfection, C666-1 and 13-9B cells were harvested, and $1 \times 10^{5}$ cells were mixed with $1 \mathrm{ml}$ RIPA solution (Sangon Biotech Co., Ltd.) to extract total proteins. The BCA method (Sigma-Aldrich; Merck KGaA) was used to measure protein concentration. Proteins were incubated with sample buffer and boiled for $5 \mathrm{~min}$ to denature the proteins. Protein samples (30 $\mu \mathrm{g}$ per lane) were subsequently loaded on a $12 \%$ gel, resolved using SDS-PAGE and transferred onto PVDF membranes. Membranes were blocked with PBS containing $5 \%$ skimmed milk at $22^{\circ} \mathrm{C}$ for $2 \mathrm{~h}$. Membranes were incubated with rabbit polyclonal primary antibodies against PTEN (1:1,200; cat. no. ab31392; Abcam) or GAPDH antibody (1:900; cat. no. ab9485; Abcam) at $4^{\circ} \mathrm{C}$ overnight, and subsequently with horseradish peroxidase-conjugated goat anti-rabbit immunoglobulin $\mathrm{G}$ secondary antibody $(1: 1,200$; cat. no. MBS435036; MyBioSource, Inc.) at $22^{\circ} \mathrm{C}$ for $2 \mathrm{~h}$. Enhanced chemiluminescence reagent (Sigma-Aldrich; Merck KGaA) was used to detect the signal on the membrane. Densitometry analysis was performed using Image $\mathbf{J}$ version 1.46 (National Institutes of Health). GAPDH was used as the loading control.

Statistical analysis. Each experiment in this study was performed three times. All data are expressed as the mean values \pm SEM. GraphPad Prism 6 (GraphPad Software, Inc.) was used for all data analysis. Differences between non-tumor 
A

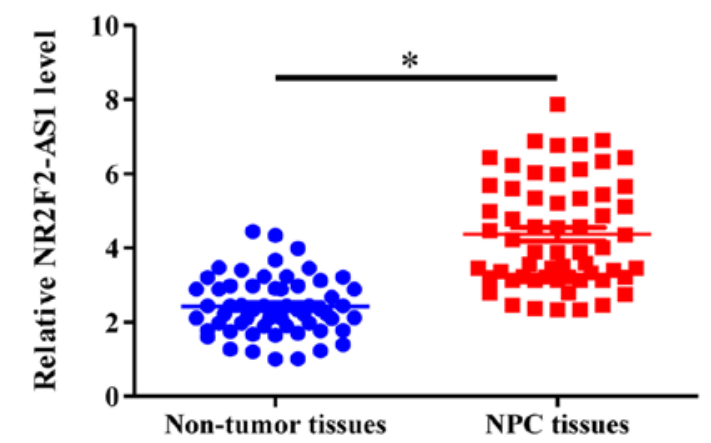

C

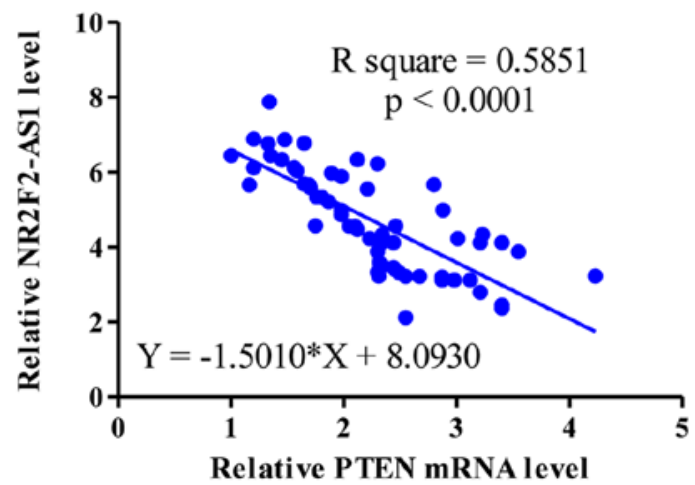

B

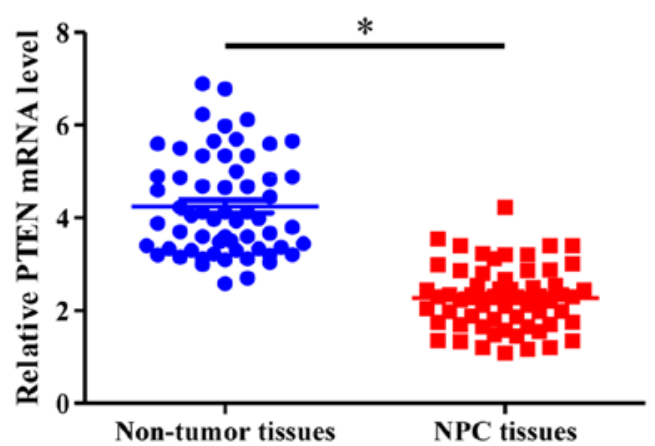

D

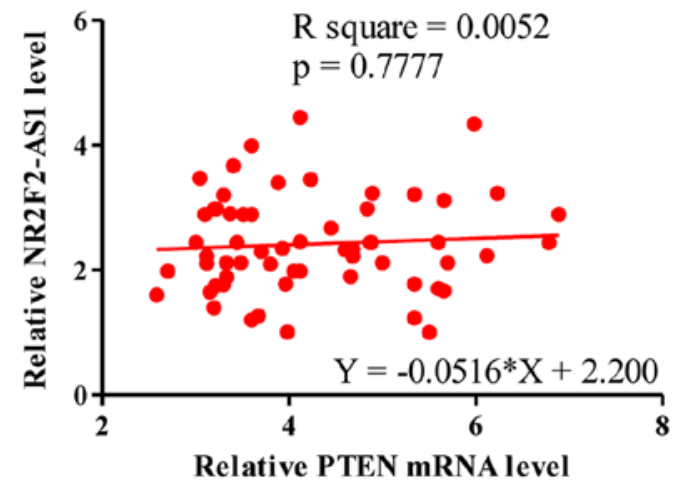

Figure 1. NR2F2-AS1 and PTEN expression levels were negatively associated in NPC tissues. Reverse transcription-quantitative polymerase chain reaction and paired t-test were used to measure and compare (A) NR2F2-AS1 and (B) PTEN expression levels in NPC tissues with non-tumor tissues (n=58). Linear regression was used to analyze the association between NR2F2-AS1 and PTEN expression levels in (C) NPC and (D) non-tumor tissues. Data are presented as the mean of three replicates. ${ }^{*} \mathrm{P}<0.05$. NPC, nasopharyngeal carcinoma. PTEN, phosphatase and tensin homolog.

and NPC tissues were compared using a Student's paired t-test. Differences in $>3$ groups were compared using an ANOVA followed by a post hoc Tukey's test. Linear regression was used for association analysis. The 58 patients with NPC were grouped into high $(n=27)$ and low $(n=31)$ groups according to NR2F2-AS1 expression levels in NPC tissues, and into high $(n=28)$ and low $(n=30)$ according to PTEN expression levels in NPC tissues, according to the Youden's index of receiver operating characteristic curve. Survival curves were plotted using the Kaplan-Meier method and compared by log-rank test. $\mathrm{P}<0.05$ was considered to indicate a statistically significant difference.

\section{Results}

NR2F2-AS1 and PTEN expression are negatively associated with each other in NPC tissues. RT-qPCR was performed to measure the expression levels of NR2F2-AS1 and PTEN in NPC and non-tumor tissues. Results were compared using a paired t-test. NPC tissues with undetectable PTEN expression were not included in this study. The results demonstrated that NR2F2-AS1 and PTEN expression levels were significantly higher (Fig. 1A) and lower (Fig. 1B), respectively, in NPC tissues compared with non-tumor tissues $(\mathrm{P}<0.05)$. Linear regression was performed to analyze the association between NR2F2-AS1 and PTEN. The results demonstrated that NR2F2-AS1 and PTEN expression levels were significantly negatively associated in NPC tissues ( $\mathrm{P}<0.0001$; Fig. 1C), which was not the case in non-tumor tissues ( $\mathrm{P}=0.7777$; Fig. 1D).

NR2F2-AS1 and PTEN expression levels can predict survival in patients with NPC. Survival curves were plotted using the Kaplan-Meier method and compared using a log-rank test. As presented in Fig. 2A, patients with high NR2F2-AS1 expression levels had a significantly lower 5-year survival rate compared with patients in the low NR2F2-AS1 expression level group $(\mathrm{P}=0.0120)$. Conversely, the overall survival rate of patients in the high PTEN expression level group was significantly higher compared with patients in the low PTEN expression level group ( $\mathrm{P}=0.0147$; Fig. 2B).

NR2F2-AS1 siRNA silencing leads to PTEN upregulation in NPC cells. C666-1 and 13-9B cells were transfected with NR2F2-AS1 siRNA. Compared with C and NC (negative control siRNA transfection) groups, expression levels of NR2F2-AS1 were significantly reduced $24 \mathrm{~h}$ post-transfection (Fig. 3A; $\mathrm{P}<0.05$ ). Furthermore, cell transfection with NR2F2-AS1 siRNA induced a significant increase in PTEN expression at the mRNA and protein levels (Fig. 3B; $\mathrm{P}<0.05$ ).

NR2F2-AS1 siRNA silencing and PTEN overexpression results in altered proliferation and apoptosis of NPC cells. Overexpression of PTEN in both C666-1 (Fig. S1A) and 13-9B (Fig. S1B) was confirmed by RT-qPCR. Cell proliferation 
A

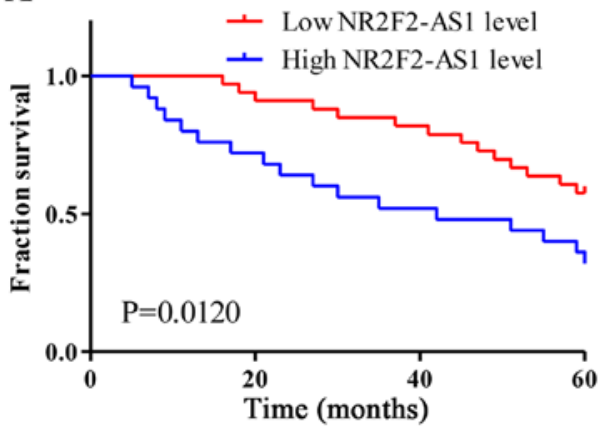

B

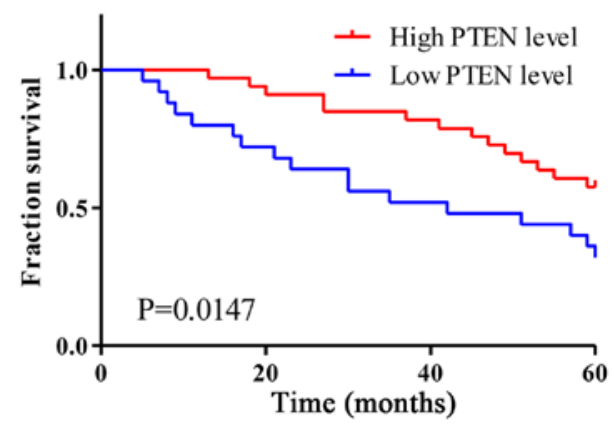

Figure 2. NR2F2-AS1 and PTEN expression in NPC tissues predicted survival. A total of 58 patients with NPC were grouped into (A) high (n=27) and low $(n=31)$ NR2F2-AS1 expression level groups and in $(B)$ high $(n=28)$ and low $(n=30)$ PTEN expression level groups according to Youden's index. Survival curves and analysis were plotted using the Kaplan-Meier method and compared using a log-rank test. NPC, nasopharyngeal carcinoma. PTEN, phosphatase and tensin homolog.

A

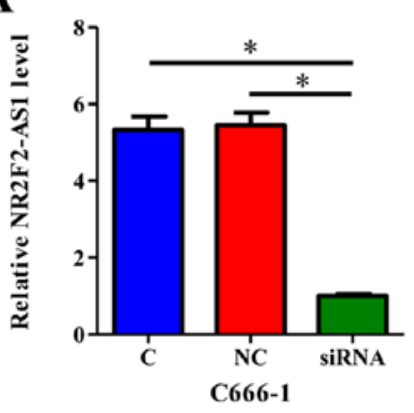

B
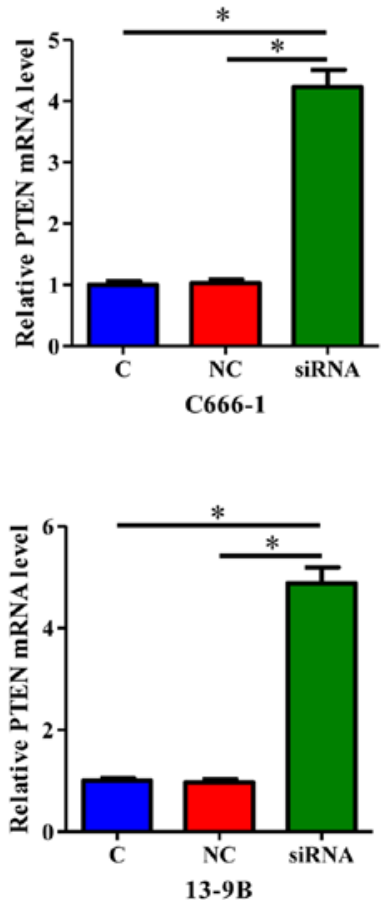
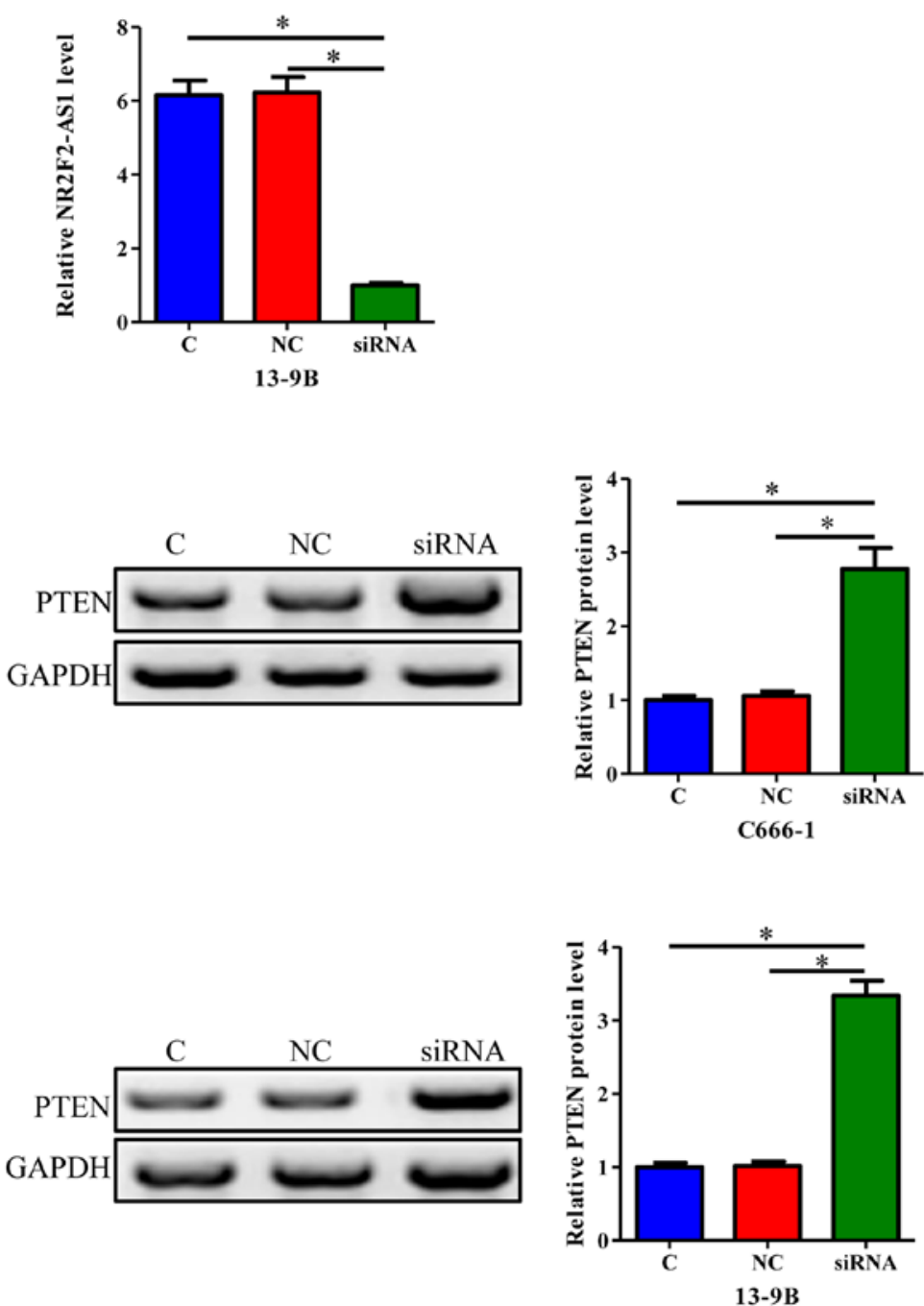

Figure 3. NR2F2-AS1 siRNA silencing led to PTEN upregulation in NPC cells. (A) NPC cells were transfected with NR2F2-AS1 and PTEN siRNAs and confirmed by RT-qPCR at 24 h post-transfection (B). Effects of NR2F2-AS1 siRNA on PTEN expression levels were analyzed by RT-qPCR and western blotting. Data were compared using an ANOVA followed by a post hoc Tukey's test. Data are presented as the mean value of three independent biological replicates. 'P<0.05. NPC, nasopharyngeal carcinoma; C, control; NC, negative control; PTEN, phosphatase and tensin homolog; RT-qPCR, reverse transcription-quantitative polymerase chain reaction; si, small interfering.

and apoptosis were analyzed following NR2F2-AS1 siRNA silencing and PTEN overexpression. The results demonstrated that, compared with $\mathrm{C}$ and NC groups, NR2F2-AS1 siRNA silencing and PTEN overexpression resulted in significantly 

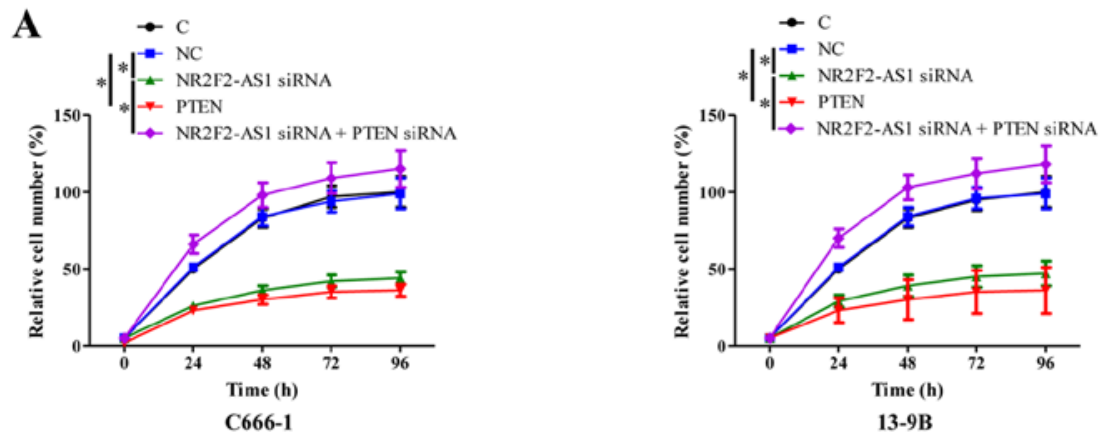

B
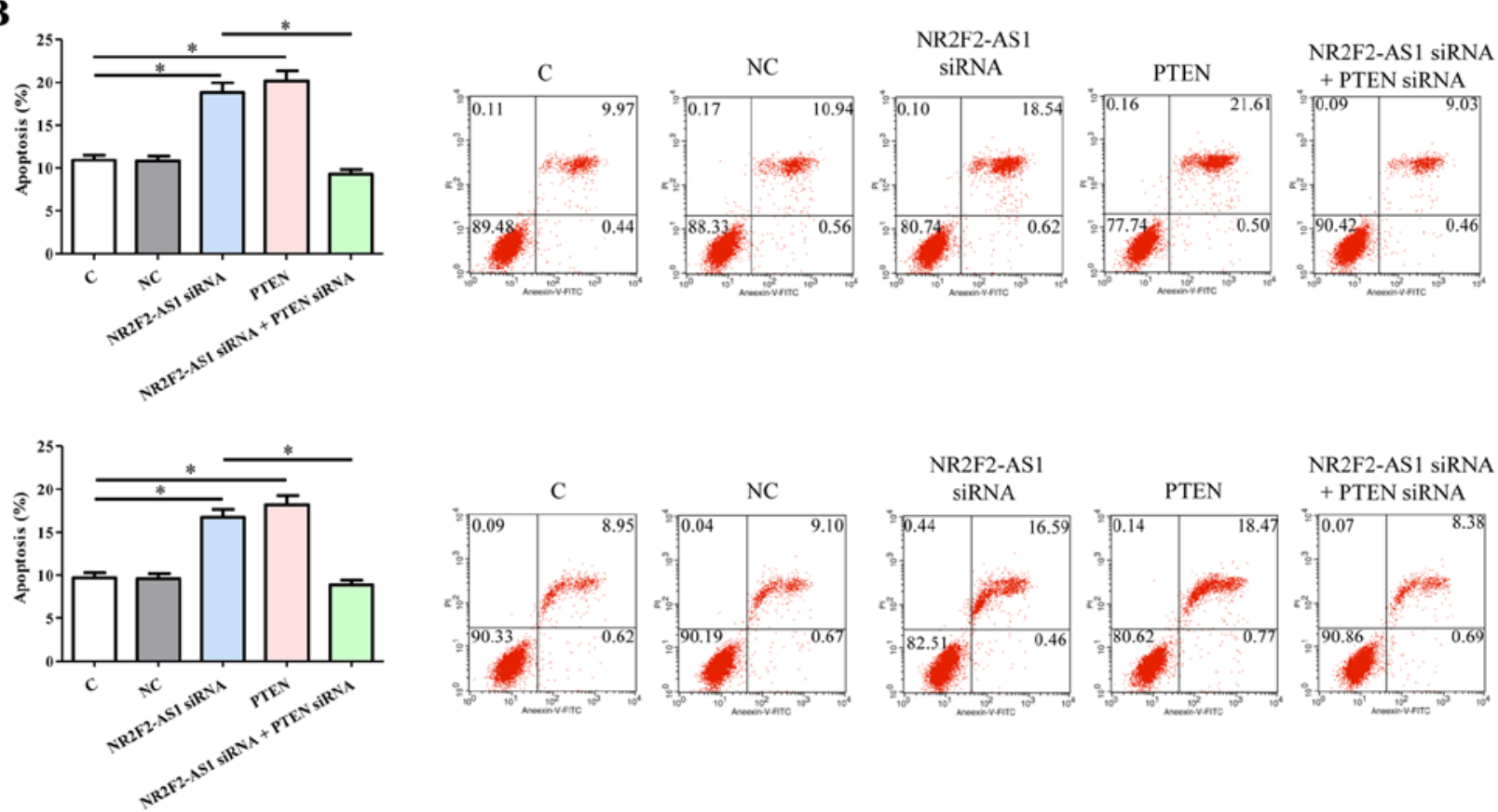

Figure 4. NR2F2-AS1 siRNA silencing and PTEN overexpression induced altered NPC cell proliferation and apoptosis. Effects of NR2F2-AS1 and PTEN siRNA silencing and of PTEN overexpression on NPC cell (A) proliferation and (B) apoptosis. Data were compared by ANOVA followed by a post hoc Tukey's test. Data are presented as the mean value of three independent biological replicates. ${ }^{\text {"P }}<0.05$. NPC, nasopharyngeal carcinoma; $\mathrm{C}$, control; NC, negative control; PTEN, phosphatase and tensin homolog; si, small interfering.

decreased proliferation (Fig. 4A), and increased apoptosis (Fig. 4B) of NPC cells. In addition, PTEN siRNA silencing completely reversed the effects of NR2F2-AS1 siRNA silencing on cell proliferation and apoptosis $(\mathrm{P}<0.05)$.

\section{Discussion}

The present study investigated the role of NR2F2-AS1 and its prognostic value in NPC. The results demonstrated that NR2F2-AS1 was upregulated in NPC. In addition, NR2F2-AS1 may affect the expression of PTEN, which is known to regulate cancer cell proliferation and apoptosis (7-9). The present study also reported that NR2F2-AS1 and PTEN expression levels may be considered as predictive biomarkers for the survival of patients with NPC.

A previous study by Khew-Goodall et al (14) reported a positive correlation between the expression levels of PTEN and of miR-320 family members in the tumor stroma of breast cancer. Furthermore, Bronisz et al (17) demonstrated that PTEN can upregulate miR-320 expression to reprogram the tumor microenvironment, by acting on the stromal fibroblasts to inactivate oncogenic secretome. miR-320 is a well-characterized tumor-suppressive miRNA with exhibits critical functions in the regulation of cancer cell behaviors, such as inhibiting cancer cell proliferation and inducing apoptosis (18). As an oncogenic lncRNA, NR2F2-AS1 in lung cancer may serve as a miRNA sponge and inhibit miR-320b (13); however, this conclusion was only made following bioinformatics analysis (13).

In the present study, a negative association between PTEN and NR2F2-AS1 expression levels in NPC tissues was observed, which was not the case in non-tumor tissues. In addition, PTEN was upregulated following NR2F2-AS1 knockdown. It has been demonstrated that downregulated expression of miR-320 members, including miR-320a, is observed in NPC (19); however, PTEN is not a target of miR-320. Furthermore, no alteration of miR-320b expression levels following NR2F2-AS1 siRNA silencing was observed (data not shown). It has been reported that PTEN activation can inhibit the PI3K/Akt pathway, which is the primary cancer cell survival pathway, and subsequently induce cancer cell apoptosis (8). NR2F2-AS1 may therefore upregulate PTEN expression, which subsequently induces apoptosis of NPC cells. In the present study, a 5-year follow-up was performed 
to analyze the association between NR2F2-AS1 and PTEN expression levels and the survival of patients with NPC. At present, early diagnosis of NPC remains limited, due to the lack of sensitive and specific markers, and insufficiency of suitable imaging techniques (20). Precise prognosis would allow the choice of appropriate treatment and care program, and therefore improve the survival of patients with NPC. The data from this study suggested that patients with NPC and high NR2F2-AS1 expression level or low PTEN expression levels exhibited reduced survival times. Therefore, improved therapeutic approaches may improve the survival times of these patients

In conclusion, the present study demonstrated that NR2F2-AS1 expression levels were upregulated in NPC and may interact with PTEN in order to participate in NPC development.

\section{Acknowledgements}

Not applicable.

\section{Funding}

No funding was received.

\section{Availability of data and materials}

The datasets used and/or analyzed during the present study are available from the corresponding author on reasonable request.

\section{Authors' contributions}

HQ designed the study, performed the experiments, analyzed the data and wrote the manuscript. CQ performed the experiments, performed the literature search and reviewed the manuscript. All authors read and approved the final version of the manuscript.

\section{Ethics approval and consent to participate}

This study was approved by the Ethics Committee of Dongying Shengli Hospital. Signed informed consent was provided by all patients prior to the study.

\section{Patient consent for publication}

Not applicable.

\section{Competing interests}

The authors declare that they have no competing interests.

\section{References}

1. Chua MLK, Wee JTS, Hui EP and Chan ATC: Nasopharyngeal carcinoma. Lancet 387: 1012-1024, 2016.

2. Chan AT, Teo PM and Johnson PJ: Nasopharyngeal carcinoma. Ann Oncol 13: 1007-1015, 2002.

3. Jiang Q, Zhou Y, Yang H, Li L, Deng X, Cheng C, Xie Y, Luo X, Fang W and Liu Z: A directly negative interaction of miR-203 and ZEB2 modulates tumor stemness and chemotherapy resistance in nasopharyngeal carcinoma. Oncotarget 7: 67288-67301, 2016.

4. Song P and Yin SC: Long non-coding RNA EWSAT1 promotes human nasopharyngeal carcinoma cell growth in vitro by targeting miR-326/-330-5p. Aging (Albany NY) 8: 2948-2960, 2016.

5. Lee AW, Ma BB, Ng WT and Chan AT: Management of nasopharyngeal carcinoma: Current practice and future perspective. J Clin Oncol 33: 3356-3364, 2015.

6. Tsang CM and Tsao SW: The role of Epstein-Barr virus infection in the pathogenesis of nasopharyngeal carcinoma. Virol Sin 30: 107-121, 2015.

7. Steck PA, Pershouse MA, Jasser SA, Yung WK, Lin H, Ligon AH, Langford LA, Baumgard ML, Hattier T, Davis T, et al: Identification of a candidate tumour suppressor gene, MMAC1, at chromosome $10 \mathrm{q} 23.3$ that is mutated in multiple advanced cancers. Nat Genet 15: 356-362, 1997.

8. Georgescu MM: PTEN tumor suppressor network in PI3K-Akt pathway control. Genes Cancer 1: 1170-1177, 2010

9. Chu EC and Tarnawski AS: PTEN regulatory functions in tumor suppression and cell biology. Med Sci Monit 10: RA235-RA241, 2004.

10. Dillon LM and Miller TW: Therapeutic targeting of cancers with loss of PTEN function. Curr Drug Targets 15: 65-79, 2014.

11. Liao Y, Shen L, Zhao H, Liu Q, Fu J, Guo Y, Peng R and Cheng L: LncRNA CASC2 interacts with miR-181a to modulate glioma growth and resistance to TMZ through PTEN pathway. J Cell Biochem 118: 1889-1899, 2017.

12. Gutschner T and Diederichs S: The hallmarks of cancer: A long non-coding RNA point of view. RNA Biol 9: 703-719, 2012.

13. Zhang S, Zhang X, Sun Q, Zhuang C, Li G, Sun L and Wang H: LncRNA NR2F2-AS1 promotes tumourigenesis through modulating BMI1 expression by targeting miR-320b in non-small cell lung cancer. J Cell Mol Med 23: 2001-2011, 2019.

14. Khew-Goodall Y and Goodall GJ: Stromal miR-320 keeps an oncogenic secretome in check. Nat Cell Biol 14: 124-125, 2012.

15. Lydiatt WM, Patel SG, O'Sullivan B, Brandwein MS, Ridge JA, Migliacci JC, Loomis AM and Shah JP: Head and neck cancers-major changes in the American Joint Committee on cancer eighth edition cancer staging manual. CA Cancer J Clin 67: 122-137, 2017.

16. Livak KJ and Schmittgen TD: Analysis of relative gene expression data using real-time quantitative PCR and the 2(-Delta Delta C(T)) method. Methods 25: 402-408, 2001.

17. Bronisz A, Godlewski J, Wallace JA, Merchant AS, Nowicki MO, Mathsyaraja H, Srinivasan R, Trimboli AJ, Martin CK, Li F, et al: Reprogramming of the tumour microenvironment by stromal PTEN-regulated miR-320. Nat Cell Biol 14: 159-167, 2011.

18. Iorio MV and Croce CM: microRNA involvement in human cancer. Carcinogenesis 33: 1126-1133, 2012.

19. Qi X, Li J, Zhou C, Lv C and Tian M: MicroRNA-320a inhibits cell proliferation, migration and invasion by targeting BMI-1 in nasopharyngeal carcinoma. FEBS Lett 588: 3732-3738, 2014.

20. Tabuchi K, Nakayama M, Nishimura B, Hayashi K and Hara A: Early detection of nasopharyngeal carcinoma. Int $\mathbf{J}$ Otolaryngol 2011: 638058, 2011.

This work is licensed under a Creative Commons Attribution-NonCommercial-NoDerivatives 4.0 International (CC BY-NC-ND 4.0) License. 